\title{
Article \\ Spatial Immunology in Liver Metastases from Colorectal Carcinoma according to the Histologic Growth Pattern
}

\author{
Gemma Garcia-Vicién ${ }^{1}\left(\mathbb{0}\right.$, Artur Mezheyeuski ${ }^{2, *}{ }^{10}$, Patrick Micke ${ }^{2} \oplus$, Núria Ruiz ${ }^{1,3}{ }^{\circledR}$, José Carlos Ruffinelli ${ }^{1,4}$, \\ Kristel Mils ${ }^{1,5}$, María Bañuls ${ }^{1}$, Natàlia Molina ${ }^{1}$, Ferran Losa ${ }^{1,4}$, Laura Lladó ${ }^{1,5}$ and David G. Molleví ${ }^{1,6, *(D)}$
}

1 Tumoral and Stromal Chemoresistance Group, Oncobell Program, Institut d'Investigacions Biomèdiques de Bellvitge (IDIBELL), Gran Via 197-203, L’Hospitalet de Llobregat, 08908 Barcelona, Catalonia, Spain; ggarciav@idibell.cat (G.G.-V.); nuria.ruiz@bellvitgehospital.cat (N.R.); jruffinelli@iconcologia.net (J.C.R.); kristel.mils@bellvitgehospital.cat (K.M.); maria.banuls@iconcologia.net (M.B.); nmolina@idibell.cat (N.M.); flosa@iconcologia.net (F.L.); laurallado@bellvitgehospital.cat (L.L.)

2 Department of Immunology, Genetics and Pathology, Uppsala University, S-75105 Uppsala, Sweden; patrick.micke@igp.uu.se

3 Department of Pathology, Hospital Universitari de Bellvitge, L'Hospitalet de Llobregat, 08908 Barcelona, Catalonia, Spain

4 Department of Medical Oncology, Institut Català d'Oncologia, L'Hospitalet de Llobregat, 08908 Barcelona, Catalonia, Spain

5 Department of Surgery, Hospital Universitari de Bellvitge, L'Hospitalet de Llobregat, 08908 Barcelona, Catalonia, Spain

check for updates

Citation: Garcia-Vicién, G.; Mezheyeuski, A.; Micke, P.; Ruiz, N.; Ruffinelli, J.C.; Mils, K.; Bañuls, M.; Molina, N.; Losa, F.; Lladó, L.; et al. Spatial Immunology in Liver Metastases from Colorectal Carcinoma according to the Histologic Growth Pattern. Cancers 2022, 14, 689. https://doi.org/ 10.3390 / cancers 14030689

Academic Editors: Alessandro Ottaiano, Michele Caraglia and Luisa Circelli

Received: 23 December 2021

Accepted: 27 January 2022

Published: 29 January 2022

Publisher's Note: MDPI stays neutral with regard to jurisdictional claims in published maps and institutional affiliations.

Copyright: (C) 2022 by the authors. Licensee MDPI, Basel, Switzerland. This article is an open access article distributed under the terms and conditions of the Creative Commons Attribution (CC BY) license (https:// creativecommons.org/licenses/by/ $4.0 /)$.
6 Program Against Cancer Therapeutic Resistance (ProCURE), Institut Català d'Oncologia, L'Hospitalet de Llobregat, 08908 Barcelona, Catalonia, Spain

* Correspondence: artur.mezheyeuski@igp.uu.se (A.M.); dgmollevi@iconcologia.net (D.G.M.); Tel.: +34-93-260-7370 (D.G.M.); Fax: +34-93-260-7466 (D.G.M.)

Simple Summary: In the era of immunotherapy, the tumor microenvironment (TME) has attracted special interest. However, colorectal liver metastases (CRC-LM) present histological peculiarities that could affect the interaction of immune and tumor cells such as fibrotic encapsulation and dense intratumoral stroma. We explored the spatial distribution of lymphocytic infiltrates in CRC-LM in the context of the histologic growth patterns using multispectral digital pathology providing data on three different scenarios, tumor periphery, invasive margin, and central tumoral areas. Our results illustrate a similar poor cell density of $\mathrm{CD}^{+}$cells between different metastases subtypes in intratumoral regions. However, in encapsulated metastases, cytotoxic cells reach the tumor cells while remaining retained in stromal areas in non-encapsulating metastases. Some aspects are still unresolved, such as understanding the reason why most lymphocytes are largely retained in the capsule.

Abstract: Colorectal cancer liver metastases (CRC-LM) present differential histologic growth patterns (HGP) that determine the interaction between immune and tumor cells. We explored the spatial distribution of lymphocytic infiltrates in CRC-LM in the context of the HGP using multispectral digital pathology. We did not find statistically significant differences of immune cell densities in the central regions of desmoplastic $\left({ }_{d} \mathrm{HGP}\right)$ and non-desmoplastic $\left({ }_{n d} \mathrm{HGP}\right)$ metastases. The spatial evaluation reported that ${ }_{\mathrm{d}} \mathrm{HGP}-$ metastases displayed higher infiltration by $\mathrm{CD} 8^{+}$and $\mathrm{CD} 20^{+}$cells in peripheral regions as well as $\mathrm{CD}^{+}$and $\mathrm{CD}_{4} 5 \mathrm{RO}^{+}$cells in ${ }_{n d} \mathrm{HGP}-$ metastases. However, the reactive stroma regions at the invasive margin (IM) of ${ }_{n d}$ HGP-metastases displayed higher density of $\mathrm{CD} 4^{+}, \mathrm{CD} 20^{+}$,

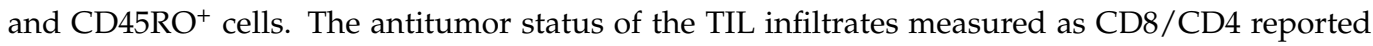
higher values in the IM of encapsulated metastases up to $400 \mu \mathrm{m}$ towards the tumor center $(p<0.05)$. Remarkably, the IM of ${ }_{\mathrm{d}} \mathrm{HGP}$-metastases was characterized by higher infiltration of $\mathrm{CD}^{+}$cells in the epithelial compartment parameter assessed with the ratio CD8 $8^{\text {epithelial }} / \mathrm{CD}^{\text {stromal }}$, suggesting anti-tumoral activity in the encapsulating lesions. Taking together, the amount of $\mathrm{CD}^{+}$cells is comparable in the IM of both HGP metastases types. However, in ${ }_{\mathrm{d}} \mathrm{HGP}$-metastases some cytotoxic cells reach the tumor nests while remaining retained in the stromal areas in ${ }_{n d}$ HGP-metastases. 
Keywords: liver metastases; lymphocyte; immunology; desmoplasia; growth pattern; multiplex

\section{Introduction}

Colorectal carcinoma (CRC) is the third most frequent tumor in the world, with $1,932,000$ new cases annually worldwide (GLOBOCAN 2020). The incidence of this tumor increases by $2 \%$ each year and is the primary cause of death due to cancer. The number of deaths annually is approximately half that of its incidence. Therefore, tackling this disease is one of the highest priority health challenges. In addition, approximately $50 \%$ of patients will develop either synchronous or metachronous colorectal liver metastases (CRC-LM) [1], with less than $10 \%$ of 5 -year survival rates in these cases [2]. Moreover, the great majority of patients with metastasis (around 70\%) are treated solely with chemotherapy, biologics in a selected group of patients [3] and immunotherapy in cases of mismatch repair deficiency [4]. Therefore, it is important to improve our knowledge of these tumors particularly for the metastatic setting.

Recent advances in immunoncology have sparked interest in the interaction of immune cells with tumor cells and other stromal cells. However, CRC-LM present histological peculiarities that could affect such interaction. CRC-LM grow according to three histological growth patterns (HGP) [5], desmoplastic $\left({ }_{d} H G P\right)$, pushing $\left({ }_{p} H G P\right)$ and replacement $\left.{ }_{\mathrm{r}} \mathrm{HGP}\right)$. Pushing and replacement subtypes are normally grouped in a single class, as non-desmoplastic (nd HGP). The desmoplastic subtype is characterized by a dense fibrotic capsule surrounding the tumors. No contact takes place between tumor cells and hepatocytes. Around $40 \%$ of CRC-LM displayed such encapsulation in at least more than $50 \%$ of its interface with the normal parenchyma and $20 \%$ displayed a complete fibrotic rim, this group of patients being those with a very good outcome [6]. This fibrotic rim is formed by cancer-associated fibroblasts (CAFs) and a dense lympho-histiocytic infiltrate and massive matrix deposition. Using conventional histology staining, the lymphocytic infiltration in ${ }_{d} \mathrm{HGP}$ is easily identifiable as a well-defined belt in the most distal half of the fibrotic rim. However, its detailed characterization, as well as the biologic reason for the lack of infiltration in more central areas of the fibrotic rim and closer to the tumor has still not been fully elucidated.

The aim of this study was to explore the spatial distribution of lymphocytic infiltrates in CRC-LM in the context of the HGPs using a multiplex immunohistochemistry (mIHC) and spatial image analysis.

\section{Materials and Methods}

\subsection{Study Cohort/Material}

Twenty-two consecutive cases of untreated CRC-LM were obtained with the approval of the Ethics Committee of the Hospital Universitari de Bellvitge (IDIBELL) after the informed consent of the patients. Thirteen cases were characterized as desmoplastic/encapsulated ( ${ }_{d} \mathrm{HGP}$ ) and nine cases as non-desmoplastic (nd HGP), characterized by the absence of the fibrotic rim between the liver parenchyma and the tumor. Five cases out of twenty-two were synchronous metastases, three characterized as ${ }_{\mathrm{d}} \mathrm{HGP}$ and two as ${ }_{n d}$ HGP. HGP was scored following previously reported consensus guidelines [7]. No MSI samples were included.

\subsection{Multiplex Immunofluorescence Staining}

Quantitative multiplex staining was performed in whole surgical FFPE sections.

Briefly, four $\mu \mathrm{m}$ thick sections were de-paraffinized, rehydrated and subjected to HIAR (microwave, $\mathrm{pH} 9,15 \mathrm{~min}$ ). To enable multiplexing, the staining procedure included several iterative cycles. In each of them the primary antibody (incubation time $30 \mathrm{~min}, \mathrm{RT}$ ) and the secondary polymerized reporter enzyme staining system (undiluted, incubation time $10 \mathrm{~min}, \mathrm{RT}$ ) were applied, and followed by tyramide signal amplification and developing 
with Opal ${ }^{\mathrm{TM}}$ fluorophore (1:100, incubation time $\left.10 \mathrm{~min}, \mathrm{RT}\right)$ (Akoya Biosciences). After a target was completed, HIAR (microwave, pH6, $15 \mathrm{~min}$ ) was applied to quench endogenous peroxidase activity, to allow both antigen retrieval and removal of antibodies and polymer system from the previous cycle. After the final staining cycle, $4^{\prime}$,6-diamidino-2phenylindole (DAPI) was applied to visualize nuclei.

A detailed procedure of retrieval buffers, primary antibodies, and amplification systems are described in supplementary material and methods.

\subsection{Imaging}

Imaging was performed with the Vectra Polaris system (Akoya Biosciences, Marlborough, MA, USA). First, each whole slide was scanned at $10 \times$ magnification. Then, the regions $(1.86 \times 1.39 \mathrm{~mm})$ for image acquisition were selected to capture areas from the central part of the metastases and from the periphery. The selected regions were imaged in a multispectral mode at a resolution of 2 pixels per $1 \mu \mathrm{m}$.

\subsection{Image Analysis, Thresholding and Immune Cell Subclasses}

Image spectral deconvolution and analysis were performed by inForm (2.4.8) software (Akoya), described in detail previously [8,9] In short, images were classified into three categories: two tissue compartments (the epithelial compartment and stroma compartment), and blank areas. DAPI nuclear staining was used to perform cell segmentation. The images were reviewed for artifacts, staining defects, and the accumulation of immune cells in necrotic areas and intraglandular structures, which were manually excluded.

Marker intensity thresholds were defined as described before [9].

Because tissue-classifier did not allow liver parenchyma to be distinguished from tumor tissue, as Pan-cytokeratin stained both tumor cells and hepatocytes, manual selection on each of the regions was performed. Thus, three tissue categories were segmented: Tumor tissue (can contain both epithelial and small regions of stroma between adjacent tumor glands), liver parenchyma (can contain both epithelial and stroma compartments), and stroma, including the fibrotic capsule in ${ }_{\mathrm{d}} \mathrm{HGP}$ metastases and some eventual stromal areas between liver and tumor cells in ${ }_{n d}$ HGP metastases.

\subsection{Spatial Analysis}

We evaluated cell distribution at the tumor periphery, the invasive margin, and central areas of metastatic tissue. These demarcations were defined as follows: tumor periphery, $100 \mu \mathrm{m}$ of the proximal liver parenchyma until the first row of tumor cells, including the capsule in ${ }_{\mathrm{d}}$ HGP CRC-LM; invasive margin, $800 \mu \mathrm{m}$ from the liver-tumor interface ( ${ }_{\text {H }} \mathrm{HGP}$ ) or capsule-tumor interface $\left({ }_{d} \mathrm{HGP}\right)$ to the deeper tumor areas; central areas, deeper and distal tumor areas from the liver parenchyma. Certain tissue category(ies) (for example, liver parenchyma and stroma) were used as 'reference tissue', while the cells of interest were identified in other tissue categories (for example, tumor tissue). Each cell of interest was defined by marker combination as described above. For each cell of interest in the tumor tissue, the nearest neighboring cell (of any subclass) in liver parenchyma or stroma was identified and the distance was measured. To simplify data visualization and analysis, the distances were split into four zones: 0 to $100 \mu \mathrm{m}, 100$ to $200 \mu \mathrm{m}, 200$ to $400 \mu \mathrm{m}$, 400 to $600 \mu \mathrm{m}$, taking as a reference two different measures (described in Figure 1B). Thus, a certain number of the cells of interest was identified in each of the zones. To normalize the cell counts, the zone area had to be assessed. For this purpose, spatial coordinates of all cells in the zone were retrieved. Further, a convex-hull algorithm was used to generate a polygon, covering the retrieved coordinates and to calculate the polygon area. Cell count was normalized per $\mathrm{mm}$ [2]. 

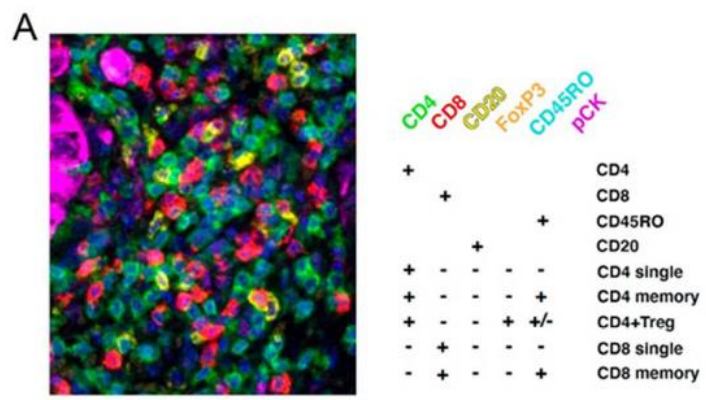

B
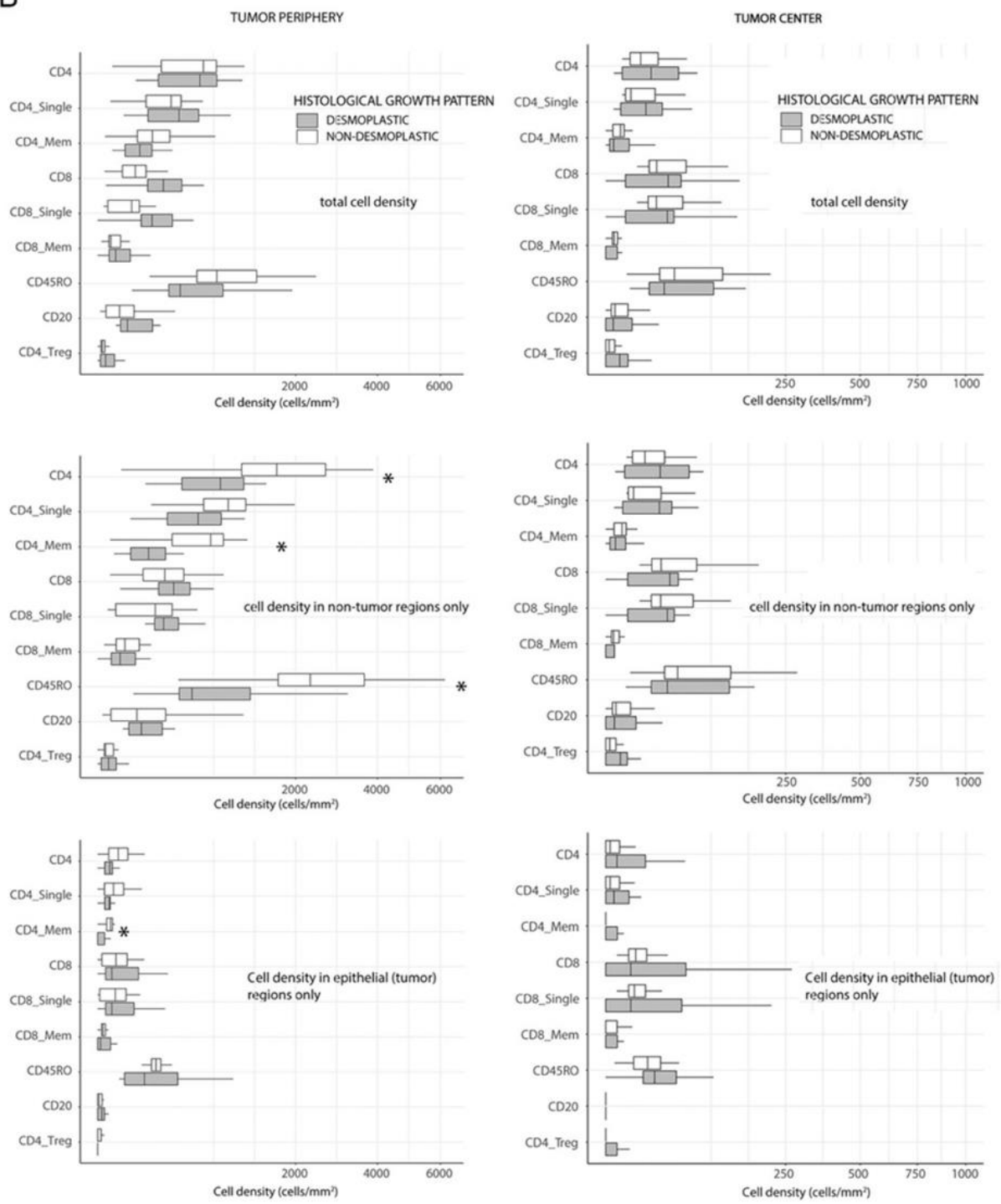

Figure 1. (A) Marker combination to identify each of the lymphocyte subsets assessed in the study. (B) Boxplots of cell densities (cells $/ \mathrm{mm}^{2}$ ) for each of the subsets assessed and stratified by HGP. We differentiated cell counts according to different topographic locations in the tumor, either in the tumor periphery or in central tumor areas as well as differentiating whether infiltrates were located in the stroma or in tumor glands. Tumor periphery was defined as the tissue fraction from $100 \mu \mathrm{m}$ of the proximal liver parenchyma until the first row of tumor cells, thus, including the capsule in dHGP CRC-LM and any rare portion of peritumoral stroma in ndHGP CRC-LM. Asterisks illustrate $p$ value $<0.05$, U-Mann Whitney test comparing dHGP vs. ndHGP. 


\subsection{Statistics}

Statistical analyses were performed using R Studio (Version 1.3.1073). The Ward algorithm was used for hierarchical clustering and the Wilcoxon signed rank test was used to compare variables between central and peripheral of the same patient. The MannWhitney U test with Pratt correction for tied data was used to compare variables between independent samples. $p<0.05$ was considered statistically significant.

\section{Results}

\subsection{The Comparison of Immune Cell Densities between Encapsulated and Non-Encapsulated} Liver Metastases

First, we aimed to compare immune infiltrating lymphocyte (TIL) densities in ${ }_{d} \mathrm{HGP}$ and ${ }_{n d}$ HGP metastases. The marker combination used to characterize TIL subclasses is displayed in Figure 1A. Because of the importance of the spatial context [10], we assessed separately the central and peripheral regions of the malignant lesions (Figure 1B). No differences were observed in central areas between metastases with different HGP (Figure 1B). Surprisingly, we also did not observe substantial differences between ${ }_{d}$ HGP and nd HGP metastases when analyzing total immune infiltrates (epithelial + stroma compartments) in peripheral regions (Figure 1B). When analyzing immune infiltrates in different compartments separately, we observed in nd HGP metastases higher infiltration of $\mathrm{CD}^{+}{ }^{+} \mathrm{CD}^{+}$ memory, $\mathrm{CD}_{45 \mathrm{RO}^{+}}$cells in stroma compartment $(p=0.041, p=0.0005$ and $p=0.032$ respectively), and $\mathrm{CD}^{+}$memory cells in the epithelial compartment $(p=0.041)$.

In conclusion, we did not find statistically significant differences between immune infiltration in central regions of metastases with different HGP. In peripheral regions nonencapsulated metastases showed higher infiltration of $\mathrm{CD}^{+}$positive lymphocyte subsets and $\mathrm{CD} 45 \mathrm{R} 0^{+}$cells.

\subsection{Spatial Assessment of TILs in Liver Metastases}

This initial analysis, however, did not take into account the differential histological characteristic represented by tumor encapsulation. Therefore, the analyzed peripheral regions in ${ }_{\mathrm{d}} \mathrm{HGP}$ were mainly represented by the capsule, which retained most of the immune infiltrates, while the peripheral regions in nd HGP, selected for analysis, mostly consisted of tumor tissue, liver parenchyma and small areas of reactive stroma. Such a difference in histological composition makes the direct comparison of peripheral regions suboptimal and suggests establishment of more reliable methods with comparable reference elements in both HGPs. With these results, we set out to evaluate lymphocyte infiltration, considering spatial location of immune infiltrates in relation to tumor and host tissue. However, the relation between these tissue elements is not equivalent in ${ }_{n d}$ HGP and ${ }_{d} H G P$. Thus, the interface between healthy and tumoral tissue is easy to identify and could serve as a reference for spatial analysis in nd HGP metastases. However, in metastases with ${ }_{\mathrm{d}} \mathrm{HGP}$ the fibrotic rim constitutes a barrier between host liver parenchyma and tumor tissue, and it is not clear whether to consider the capsule as an element of the host or the tumor. Taking into account this histological characteristic, we considered two interface lines between different tissue types, to serve as references for spatial analysis of the immune infiltrates, allowing two measurement types: A and B, as illustrated in Figure 2. Measure A evaluates cell distance from the interface between liver parenchyma and either capsule (in ${ }_{\mathrm{d}} \mathrm{HGP}$ ) or tumor (in ${ }_{n d} \mathrm{HGP}$ ) or small areas of reactive stroma (in ${ }_{\text {nd }} \mathrm{HGP}$ ). Measure A thus includes the cells, located in the capsule in ${ }_{d}$ HGP metastases, in reactive stroma in ${ }_{n d}$ HGP metastases, and in tumor tissue in both HGP. Measure B excludes cells from stroma between liver parenchyma and tumor and includes only the cells from tumor tissue, thus, strictly speaking that which corresponds to the invasive margin of the tumor. 

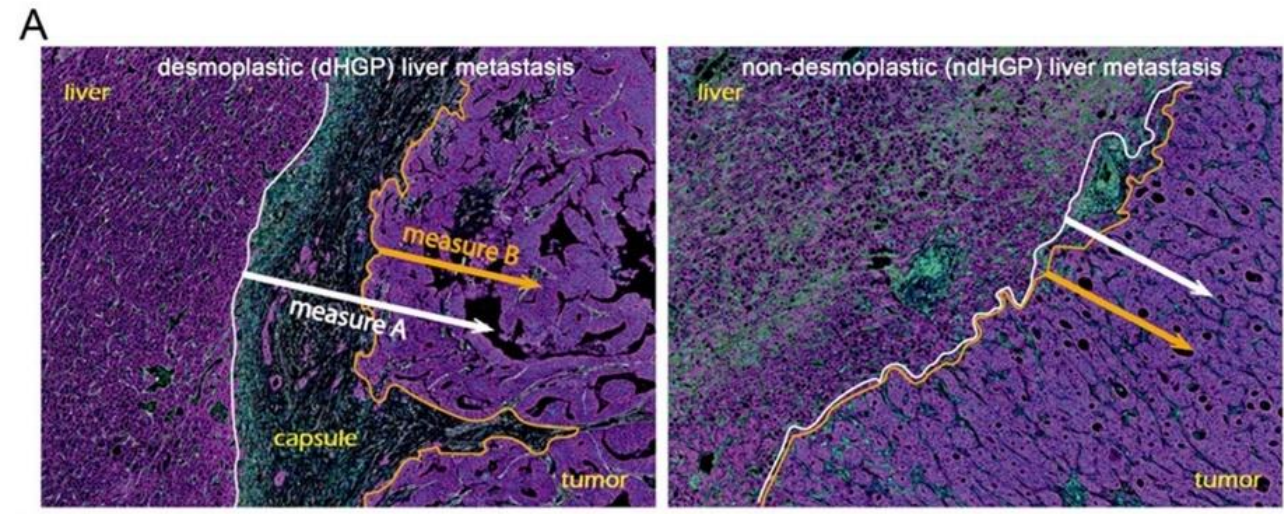

B

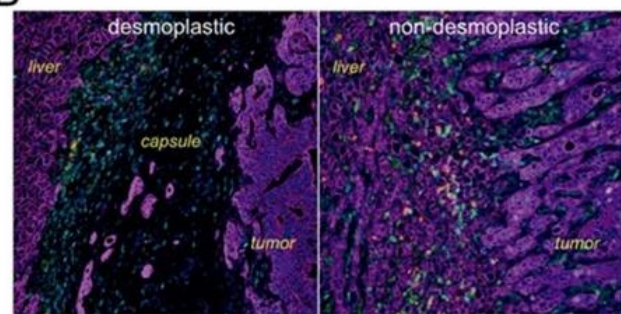

Liver-Tumor interface

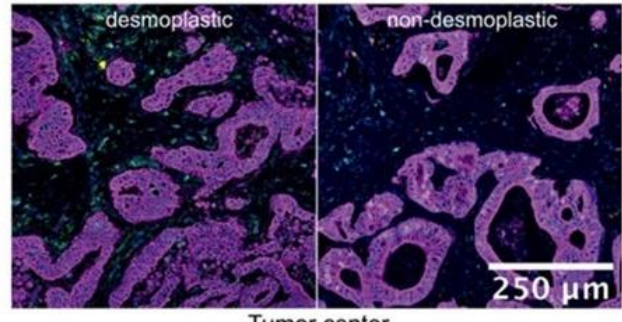

Tumor center

Figure 2. (A) Distances considered in relation to the tumor-host interface. Measure A, using as a reference the tumor-liver interface, including as part of the tumor, the fibrous capsule in case of ${ }_{\mathrm{d}} \mathrm{HGP}$ or any small stromal area in the case of ${ }_{n d}$ HGP; Measure B, using the tumor margin as a reference and then, not considering capsule or any peritumoral stromal. We use this measure throughout the manuscript to refer to the invasive margin. (B) Detailed magnification of the tumor/liver interface and central tumoral areas for both main histologic growth patterns.

Considering Measure A, desmoplastic ${ }_{\mathrm{d}} \mathrm{HGP}$ metastases displayed higher infiltration of $\mathrm{CD}^{+}, \mathrm{CD}^{+}$single positive, and $\mathrm{CD} 20^{+}$lymphocytes, particularly in areas close to the liver parenchyma (Figure 3A, left panel). This distance, in encapsulated metastases, corresponds mainly to the outer capsule fragment closest to the liver, where a large quantity of TILs is retained. However, when moving away from the parenchyma towards the tumor, the differences between HGP became less prominent and at $400 \mu \mathrm{m}$ were completely lost. These, deeper regions most likely corresponded to invasive margin in ${ }_{\mathrm{d}} \mathrm{HGP}$ and deeper tumoral regions in ${ }_{n d}$ HGP.

On the other hand, the results were different when we used Measure B. In this case, $\mathrm{CD}^{+}, \mathrm{CD}^{+}$single positive, $\mathrm{CD} 4^{+}$memory, $\mathrm{CD} 4$-Treg $\left(\mathrm{CD}^{+}{ }^{+}\right.$and $\left.\mathrm{FoxP}^{+}\right)$, and $\mathrm{CD} 45 \mathrm{R} 0^{+}$ TILs were significantly more abundant in non-encapsulated metastases (Figure $3 \mathrm{~A}$, right panel), while no difference was seen for $\mathrm{CD}^{+}$subsets.

Interestingly, the overall cell density for most of the immune subsets was lower when using Measure B not only in encapsulated metastases (which is because the majority of the immune cells in this HGP is retained in the capsule) but also in non-encapsulated metastases, probably, revealing the impact of the regions of reactive stroma, present on the tumor/liver border in non-encapsulated lesions (as an example, the infiltrates located between the white and orange lines in Figure $1 \mathrm{~B}$ right image). This difference was especially prominent for all $\mathrm{CD} 4^{+}$subsets, CD20 cells, and CD45R $0^{+}$cells.

Even larger prevalence of $\mathrm{CD}^{+}, \mathrm{CD}^{+}$single positive, $\mathrm{CD} 4^{+}$memory, $\mathrm{CD} 4$-Treg, and $\mathrm{CD} 45 \mathrm{R}^{+}$cells in terms of statistical significance and absolute cell counts was demonstrated in non-encapsulated metastases. Additionally, CD20 $0^{+}$cells showed higher levels in non-encapsulated samples, thus, demonstrating cellular composition in the regions of reactive stroma. 
A

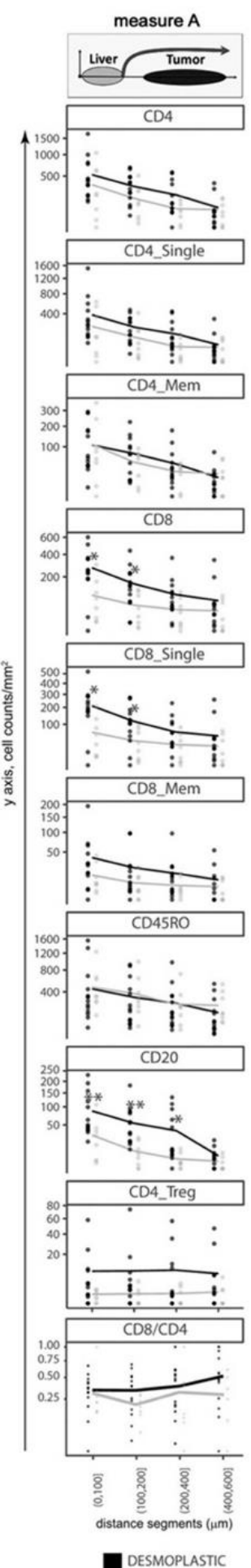

B
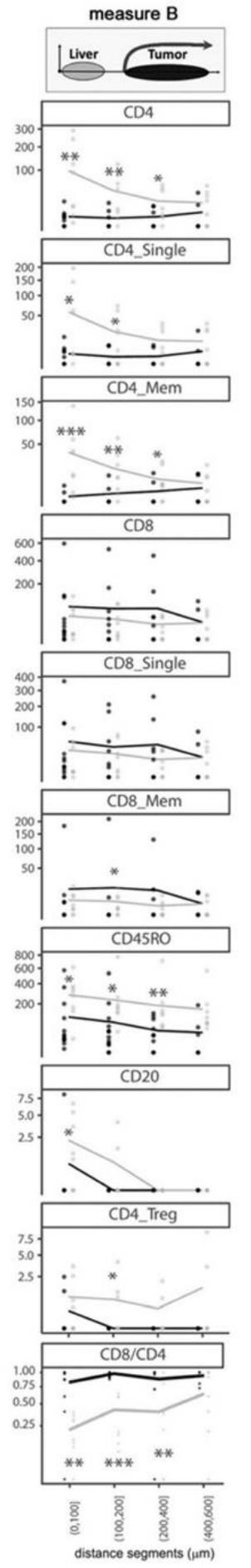

CD4_Single

CD4_Mem

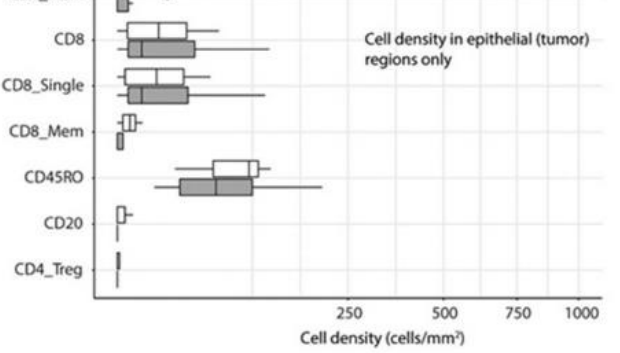

Figure 3. (A) Spatial assessment of lymphocyte subsets in ${ }_{d}$ HGP and ${ }_{n d}$ HGP CRC-LM according to two different measures, Measure A, from liver margin, Measure B, from the outer tumoral region (invasive margin). Lines show mean levels of cell densities. Dots show cell count $/ \mathrm{mm}^{2} . X$-axis is square-root transformed (distant segments, $\mu \mathrm{m}$ ). Mann-Whitney $U$ test was applied for statistical analysis. (B) Boxplots of cell densities (cells $/ \mathrm{mm}^{2}$ ) for each of the subsets were assessed and stratified by HGP according to the invasive margin. Asterisks illustrate $p$ value $<0.05$, Mann-Whitney U test comparing ${ }_{d} \mathrm{HGP}$ vs. nd HGP. 
In conclusion, the fibrotic capsule in encapsulated metastases, especially the outer capsule part is characterized by increased quantity of $\mathrm{CD}^{+}$positive lymphocyte subsets and $\mathrm{B}$ cells, in comparison to peripheral regions in nd HGP metastases. However, the invasive margin of tumor tissue in encapsulated metastases did not demonstrate enrichment of these immune cell subsets, and conversely, showed a lower density of $\mathrm{CD}^{+}$positive lymphocyte subsets and $\mathrm{CD} 45 \mathrm{R}^{+}$cells. The reactive stroma regions in non-encapsulated metastases are characterized by accumulation of $\mathrm{CD}^{+}$positive lymphocyte subsets, $\mathrm{CD} 45 \mathrm{R} 0^{+}$cells, and CD20 cells.

\subsection{The Comparison of Immune Cell Densities in the Invasive Margin of Liver Metastases}

Spatial analysis revealed the importance of accurate assessment of the histological peculiarities of liver metastases with different HGP. Because we assumed that direct interaction between immune cells and malignant tissue is of major importance for immune surveillance in metastatic disease, we focused our next analyses on the metrics, derived using 'Measure B' approach and performed manual selection of the invasive margin regions (i.e., tumor regions, excluding capsule, liver parenchyma, and reactive stroma) in both HGP.

Non-encapsulated metastases displayed higher quantity of total $\mathrm{CD}^{+}, \mathrm{CD}^{+}$single positive, $\mathrm{CD}^{+}$memory cells, as well as CD20 ${ }^{+} \mathrm{B}$ cells in the invasive margin (Figure $3 \mathrm{~B}$; $p=0.011, p=0.038, p=0.008$ and $p=0.032$ respectively). These differences corresponded mainly to inflammatory infiltrates circumscribed to the stromal compartment, which retained most of the identified infiltrates (Figure 3B, middle panel), with the exception of total $\mathrm{CD}^{+}$memory cells that demonstrated greater infiltration also in the epithelial compartment in non-encapsulated metastases (Figure 3B, lower panel).

In conclusion, the analysis of the invasive margin confirmed the spatial analysis and demonstrated that non-encapsulated metastases have higher infiltration of several CD4 ${ }^{+}$ positive lymphocyte subsets.

\subsection{The Comparison of Immune Cell Densities in the Invasive Margin and Tumor Center}

Next, we compared the inflammatory infiltrates between the invasive margin and central areas of the tumor in each sample in a pairwise manner. ${ }_{d} H G P$ displayed higher infiltration by $\mathrm{CD}^{+}{ }^{+}$total, $\mathrm{CD} 4^{+}$single positive, and CD4_Treg cells in central areas compared to the invasive margin while the trend was the opposite for these cell subtypes in

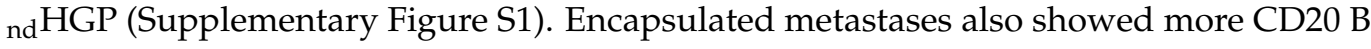
cells in central areas than in the invasive margin.

Thus, summarizing the results from two HGPs and two locations (central and invasive margin) we can state that the poorest immune infiltration is observed in the invasive margin of encapsulated metastases, while their central parts as well as the invasive margin and central parts of non-encapsulated metastases demonstrated comparable quantities of TILs.

\subsection{Anti-Tumoral Status of the Immune Infiltrates in the Invasive Margin and Tumor Center}

Our results did not match the established paradigm of TIL infiltration in tumors. Thus, TIL infiltration is expected to be associated with improved survival. Encapsulated metastases are also known to have substantially better prognosis than non-encapsulated. However, in our study, we observed lower TIL levels in malignant tissues of encapsulated metastases. We hypothesized, that such results may be explained by the presence of TILs with immune-suppressive functions. In fact, while CD8 lymphocytes are considered to be main tumor cell killers [11], among the $\mathrm{CD}^{+} \mathrm{T}$ helper subsets, the cells of type 2 (Th2) may dominate in tumors [12]. Th2 cells drive the polarization of macrophages towards M2-type and eventually create an immune-suppressive tumor microenvironment [13].

Thus, we sought to evaluate the overall anti-tumoral status of the TIL infiltrates, assuming that $\mathrm{CD}^{+}$cells represent anti-tumor immunity while $\mathrm{CD} 4^{+}$cell subsets may contain significant fraction of Th2 T cells and therefore reflect pro-tumor characteristics. To assess TIL antitumoral status we generated a metric: $\frac{\mathrm{CD} 8}{\mathrm{CD} 8+\mathrm{CD} 4}$, which is denoted further in the text as 'CD8/CD4 ratio'. 
The CD8/CD4 ratio was higher in the invasive margin, than in the central areas in ${ }_{\mathrm{d}} \mathrm{HGP}$ samples, while in nd HGP metastases the result was the opposite (Supplementary Figure S1). Further, when applying spatial analysis using Measure B, i.e., excluding any peritumoral stroma, encapsulated metastases demonstrated a higher CD8/CD4 ratio at the tumor margin and up to $400 \mu \mathrm{m}$ towards the tumor center $(p<0.05)$ (Figure $3 \mathrm{~A}$, right panel). At the same time, no difference was seen in the CD8/CD4 ratio between the central areas of the two HGP (Figure 4, upper panel). Finally, analysis of invasive margin regions confirmed the observations of spatial distribution and demonstrated a greater CD8/CD4 ratio in encapsulated metastases (Figure 4, upper panel).
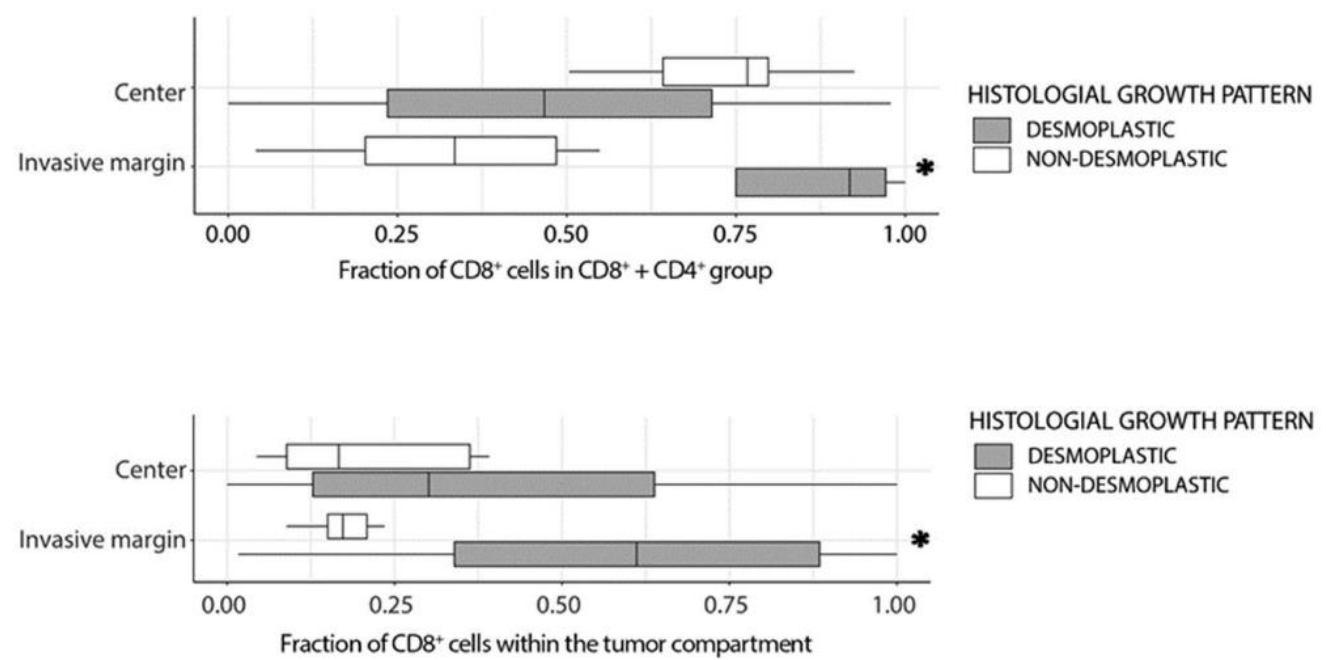

Figure 4. Top panel, boxplot for CD8/CD4 ratio comparing ${ }_{d} \mathrm{HGP}$ and ${ }_{n d} \mathrm{HGP}$ in central tumoral areas and at the invasive margin. Bottom panel, boxplot displaying the fraction of $\mathrm{CD}^{+}$cells within the epithelial compartment, both at the invasive margin and central tumor areas. Asterisks illustrate $p$ value $<0.05$, Mann-Whitney U test comparing ${ }_{d}$ HGP vs. ${ }_{n d}$ HGP.

The active anticancer response by cytotoxic cells requires their presence in the immediate vicinity of the malignant cells, enabling their direct contact and cytotoxic function. Thus, if the CD8/CD4 ratio reflects the antitumoral immune microenvironment in ${ }_{\mathrm{d}} \mathrm{HGP}$ metastases, one would expect a higher number of CD8 (cytotoxic) cells in the epithelial com-

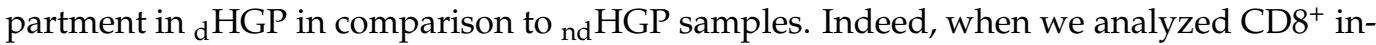
filtration in the invasive margin specifically in the epithelial compartment, in relation to the cytotoxic cells that remain limited in the stromal compartment, the encapsulated metastases demonstrated significantly higher levels (evaluated as $\frac{\text { CD8 epithelial }}{\text { CD8 epithelial+CD8 stromal }}$; Figure 4, lower panel; $p=0.021$, Mann-Whitney U test).

Collectively, the CD8/CD4 ratio, which we consider as a surrogate metric of the antitumoral status of the TIL infiltrates, was significantly elevated in the invasive margin in encapsulated metastases, in comparison to central areas and to the invasive margin in nonencapsulated lesions, potentially reflecting an anti-tumoral immune microenvironment in ${ }_{\mathrm{d}}$ HGP metastases. Moreover, the invasive margin in encapsulated metastases was characterized by higher prevalence of $\mathrm{CD}^{+}$cells in the epithelial compartment, suggesting the presence of an anti-tumoral microenvironment in ${ }_{\mathrm{d}} \mathrm{HGP}$ lesions and the presence of an immune-suppressive, pro-tumoral microenvironment in ${ }_{n d}$ HGP lesions.

\section{Discussion}

The present study aimed to characterize the spatial distribution of lymphocytic infiltrates in chemonaive CRC-LM in the context of the particular HGP. We analyzed the immune infiltrates in 22 untreated CRC-LM taking into account the characteristic HGP of metastases. We assessed the spatial distribution in the tissue on three different levels: (a) by distinguishing the tumor central and peripheral regions and the invasive margin, 
(b) by accurate measurement of immune cell distribution in relation to different histological hallmarks and (c) by the analysis of the immune infiltration in stromal and epithelial compartments. To the best of our knowledge, this is the first study, providing a systematic and reproducible approach for the topographic distribution of lymphocytic infiltrates in CRC-LM in the context of the HGP.

There are many studies related to prognostic factors in CRC-LM, whether surgical parameters [2], histopathological [14], molecular [15] or immunological parameters [16]. However, the importance of immunological parameters, such as Immunoscore [17], has recently become evident, adding to the already known predictive value of Kras mutations [18].

On the other hand, the HGP have a deep impact on the survival of patients with CRC-LM [19-21]. Likewise, the tumor microenvironment (TME) is of paramount relevance in the formation of such growth patterns, since in the fibrotic rim and the lymphocytic belt there are contained demarcate histologic structures that might define the immune response against the tumor. However, the retention on the outer portion of the capsule of such a large number of infiltrates and the association with a better prognosis remains to be elucidated.

In addition, it has already been published that lymphocytic infiltrates are not uniformly distributed in CRC-LM [22], and quite often immune cells tend to be located in the peripheral areas of the tumor [23-25]. However, in the majority of these published studies neither the HGP nor the spatial distribution was taken into consideration. More recently, Berthel A et al. [26], reported distinct patterns of immune cell infiltration in CRC-LM, revealing a certain degree of infiltrate heterogeneity depending on the distance from the invasive margin. However, no mention was made of HGP. Hoppener D et al. [27] reported that encapsulating metastases are characterized by an increase in cell densities of different cytotoxic populations, and the authors attribute this fact to the better survival of patients with these metastases. Following this observation, some authors suggested that nd HGP CRC-LM should be considered as immune desert tumors and ${ }_{\mathrm{d}} \mathrm{HGP}$ as immune excluded [28].

The main difference in our exploratory study compared to previously reported results is that we introduced a standardized reproducible approach for spatial analysis in three different locations, (i) in central areas of the tumor, where there is usually an enrichment in a mature stroma with low tumor cellularity, (ii) in the tumor invasive margin, that is, in the outermost area with the presence of malignant cells, either in contact with hepatocytes $\left({ }_{n d} H G P\right)$, reactive stroma regions $\left(n_{d} H G P\right)$, or with the fibrous capsule $\left({ }_{d} H G P\right)$ and iii) in the peripheral area, also referred to as the outer invasive margin [29], which in CRC-LM includes the capsule $\left({ }_{d} \mathrm{HGP}\right)$, reactive stroma regions $\left(\mathrm{n}_{\mathrm{d}} \mathrm{HGP}\right)$, any small portions $(100 \mu \mathrm{m})$ of peritumoral stroma, and normal liver parenchyma and small portions $(100 \mu \mathrm{m})$ of tumor.

In our initial analysis we evaluated immune cells in peripheral areas, which were not stringently defined and observed the expected prevalence of cytotoxic cells in encapsulated metastases, thus, supporting the immune excluded/immune desert paradigm. However, we noticed that most of the immune cells of the peripheral regions in ${ }_{\mathrm{d}} \mathrm{HGP}$ are retained in the outer part of the desmoplastic capsule and thus, never establish contacts with cancer cells, while most of the immune cells in ${ }_{n d}$ HGP are localized in regions of reactive stroma. When we excluded these regions form the analysis, we observed higher immune cell densities in ${ }_{n d}$ HGP lesions. Thus, we doubt that the immune excluded/immune desert concept is applicable for CRC-LM.

Interesting, the higher immune cell densities in the invasive margin of ${ }_{n d}$ HGP lesions were represented by the subsets of $\mathrm{CD}^{+}$cells. Although we could not dissect the functional characteristic of these subsets, we hypothesize they may contain an increased fraction of Th2-type T helpers with immune-suppressive capacities.

This hypothesis is supported by the analysis of the CD8/CD4 ratio, which was significantly lower in non-desmoplastic tumors, and even further confirmed by the analysis of $\mathrm{CD}^{+}$cells, which were enriched in the epithelial compartment in the invasive margin in desmoplastic tumors, suggesting cytotoxic activity. Additionally, a higher CD8/CD4 ratio was measured in the central areas of ${ }_{n d} \mathrm{HGP}$ compared to their matched invasive 
margin, which could be closely related to the mechanism of CD8 siphoning described very recently [30].

Taken together, the quantity of cytotoxic $\mathrm{CD} 8^{+}$cells is comparable in invasive margins in both HGP, but the immune microenvironment in ${ }_{n d} \mathrm{HGP}$ seem to be immune-suppressive and blocks the cytotoxic activity of $\mathrm{CD}^{+}$cells. One might therefore speculate that ${ }_{\text {nd }} \mathrm{HGP}$ CRC-LM could benefit from immune-checkpoint therapy, which may help to re-activate pre-existing antitumoral lymphocytes. On the other hand, ${ }_{d}$ HGP CRC-LM could be suitable candidates for immunotherapy based on adoptive $\mathrm{T}$ cell transfer since small amounts of cytotoxic CD8 T cells reached the tumor nests.

\section{Conclusions}

As a summary, the present study provides a description of different patterns of lymphocyte infiltration depending on the HGP. The composition and the topographic demarcation in relation to the invasive margin depicted different immune scenarios according to the HGP in CRC-LM. Perhaps the most relevant finding was the presence of immune-suppressive microenvironment in metastases with ${ }_{n d} \mathrm{HGP}$, and antitumoral immune microenvironment in metastases with ${ }_{\mathrm{d}} \mathrm{HGP}$, a fact that could explain the better prognosis of encapsulating metastases. Despite this difference, both HGP are characterized by low levels of cytotoxic lymphocytes and thus, could benefit from immune-therapies. Some aspects remain unresolved for now, such as understanding the reason for the retention of a large number of lymphocytic infiltrates in the external part of the capsule.

Supplementary Materials: The following supporting information can be downloaded at: https: / / www.mdpi.com/article/10.3390/cancers14030689/s1, Figure S1: Pairwise comparison of immune cell densities in the invasive margin (IM) and central areas of the tumor (CT). Wilcox-on signed-rank test with Pratt modification was used to look for statistically significant differences between the two different areas.

Author Contributions: J.C.R., K.M., F.L., L.L. and N.R. selected the cases, provided samples, and assessed the histologic growth pattern. G.G.-V. performed the laboratory procedures and contributed to the manuscript writing. N.M. and M.B. provided laboratory technical support. P.M. contributed with logistical support and authorization of the use of the facilities (Vectra Polaris) at Uppsala University. A.M. performed data analyses and manuscript writing. D.G.M. conceived and designed the study, provided funding support and manuscript writing. All authors have read and agreed to the published version of the manuscript.

Funding: This work was supported by grant PI18/1140 from the Fondo de Investigaciones Sanitarias of the Spanish Government, Fondo Europeo de Desarrollo Regional (FEDER) "Una manera de hacer Europa" /"A way of shaping Europe" and AGAUR grant number SGR771. In addition, GGV is recipient of the 2019 FI_B 00673 grant, funded by AGAUR the Department of Health of the Generalitat de Catalunya by the call "Ajuts per a la contractació de personal investigador novell FI 2019". We thank CERCA Programme/Generalitat de Catalunya for institutional support.

Institutional Review Board Statement: The study was conducted in accordance with the Declaration of Helsinki, and approved by the Institutional Review Board of IDIBELL (protocol code PR305/19).

Informed Consent Statement: Informed consent was obtained from all subjects involved in the study.

Data Availability Statement: The data presented in this study are available in this article (and Supplementary Material).

Conflicts of Interest: None of the authors have any conflict of interest to declare.

\section{References}

1. Tzeng, C.W.; Aloia, T.A. Colorectal liver metastases. J. Gastrointest. Surg. 2013, 17, 195-201. [CrossRef] [PubMed]

2. Adam, R.; De Gramont, A.; Figueras, J.; Guthrie, A.; Kokudo, N.; Kunstlinger, F.; Loyer, E.; Poston, G.; Rougier, P.; Rubbia-Brandt, L.; et al. The oncosurgery approach to managing liver metastases from colorectal cancer: A multidisciplinary international consensus. Oncologist 2012, 17, 1225-1239. [CrossRef] [PubMed] 
3. Van Cutsem, E.; Tabernero, J.; Lakomy, R.; Prenen, H.; Prausová, J.; Macarulla, T.; Ruff, P.; Van Hazel, G.A.; Moiseyenko, V.; Ferry, D.; et al. Addition of aflibercept to fluorouracil, leucovorin, and irinotecan improves survival in a phase III randomized trial in patients with metastatic colorectal cancer previously treated with an oxaliplatin-based regimen. J. Clin. Oncol. 2012, 30, 3499-3506. [CrossRef] [PubMed]

4. Johnsson, A.; Hagman, H.; Frodin, J.E.; Berglund, Å.; Keldsen, N.; Fernebro, E.; Sundberg, J.; Christensen, R.D.P.; Spindler, K.L.G.; Bergström, D.; et al. A randomized phase III trial on maintenance treatment with bevacizumab alone or in combination with erlotinib after chemotherapy and bevacizumab in metastatic colorectal cancer: The Nordic ACT Trial. Ann. Oncol. 2013, 24, 2335-2341. [CrossRef] [PubMed]

5. Van den Eynden, G.G.; Bird, N.C.; Majeed, A.W.; Van Laere, S.; Dirix, L.Y.; Vermeulen, P.B. The histological growth pattern of colorectal cancer liver metastases has prognostic value. Clin. Exp. Metastasis 2012, 29, 541-549. [CrossRef]

6. Galjart, B.; Nierop, P.M.H.; van der Stok, E.P.; van den Braak, R.R.C.; Höppener, D.J.; Daelemans, S.; Dirix, L.Y.; Verhoef, C.; Vermeulen, P.B.; Grünhagen, D.J. Angiogenic desmoplastic histopathological growth pattern as a prognostic marker of good outcome in patients with colorectal liver metastases. Angiogenesis 2019, 22, 355-368. [CrossRef]

7. van Dam, P.J.; van der Stok, E.P.; Teuwen, L.A.; Van den Eynden, G.G.; Illemann, M.; Frentzas, S.; Majeed, A.W.; Eefsen, R.L.; Van Den Braak, R.R.; Lazaris, A.; et al. International consensus guidelines for scoring the histopathological growth patterns of liver metastasis. Br. J. Cancer 2017, 117, 1427-1441. [CrossRef]

8. Mezheyeuski, A.; Bergsland, C.H.; Backman, M.; Djureinovic, D.; Sjöblom, T.; Bruun, J.; Micke, P. Multispectral imaging for quantitative and compartment-specific immune infiltrates reveals distinct immune profiles that classify lung cancer patients. $J$. Pathol. 2018, 244, 421-431. [CrossRef]

9. Lundgren, S.; Elebro, J.; Heby, M.; Nodin, B.; Leandersson, K.; Micke, P.; Jirström, K.; Mezheyeuski, A. Quantitative, qualitative and spatial analysis of lymphocyte infiltration in periampullary and pancreatic adenocarcinoma. Int. J. Cancer 2020, 146, 3461-3473 [CrossRef]

10. Fu, T.; Dai, L.J.; Wu, S.Y.; Xiao, Y.; Ma, D.; Jiang, Y.Z.; Shao, Z.M. Spatial architecture of the immune microenvironment orchestrates tumor immunity and therapeutic response. J. Hematol. Oncol. 2021, 14, 98. [CrossRef]

11. Raskov, H.; Orhan, A.; Christensen, J.P.; Gögenur, I. Cytotoxic CD8(+) T cells in cancer and cancer immunotherapy. Br. J. Cancer 2021, 124, 359-367. [CrossRef] [PubMed]

12. Coussens, L.M.; Zitvogel, L.; Palucka, A.K. Neutralizing tumor-promoting chronic inflammation: A magic bullet? Science 2013, 339, 286-291. [CrossRef] [PubMed]

13. Pollard, J.W. Trophic macrophages in development and disease. Nat. Rev. Immunol. 2009, 9, 259-270. [CrossRef] [PubMed]

14. Hoppener, D.J.; Galjart, B.; Nierop, P.M.H.; Buisman, F.E.; van der Stok, E.P.; Coebergh van den Braak, R.R.; van Amerongen, M.J.; Balachandran, V.P.; Jarnagin, W.R.; Kingham, T.P.; et al. Histopathological Growth Patterns and Survival After Resection of Colorectal Liver Metastasis: An External Validation Study. JNCI Cancer Spectr. 2021, 5, pkab026. [CrossRef] [PubMed]

15. Schlicker, A.; Ellappalayam, A.; Beumer, I.J.; Snel, M.H.; Mittempergher, L.; Diosdado, B.; Dreezen, C.; Tian, S.; Salazar, R.; Loupakis, F.; et al. Investigating the concordance in molecular subtypes of primary colorectal tumors and their matched synchronous liver metastasis. Int. J. Cancer 2020, 147, 2303-2315. [CrossRef]

16. Tanis, E.; Julie, C.; Emile, J.F.; Mauer, M.; Nordlinger, B.; Aust, D.; Roth, A.; Lutz, M.P.; Gruenberger, T.; Wrba, F.; et al. Prognostic impact of immune response in resectable colorectal liver metastases treated by surgery alone or surgery with perioperative FOLFOX in the randomised EORTC study 40983. Eur. J. Cancer 2015, 51, 2708-2717. [CrossRef]

17. Pages, F.; Mlecnik, B.; Marliot, F.; Bindea, G.; Ou, F.S.; Bifulco, C.; Lugli, A.; Zlobec, I.; Rau, T.T.; Berger, M.D.; et al. International validation of the consensus Immunoscore for the classification of colon cancer: A prognostic and accuracy study. Lancet 2018, 391, 2128-2139. [CrossRef]

18. Van Cutsem, E.; Cervantes, A.; Adam, R.; Sobrero, A.; Van Krieken, J.H.; Aderka, D.; Aguilar, E.A.; Bardelli, A.; Benson, A.; Bodoky, G.; et al. ESMO consensus guidelines for the management of patients with metastatic colorectal cancer. Ann. Oncol. 2016, 27, 1386-1422. [CrossRef]

19. Nielsen, K.; Rolff, H.C.; Eefsen, R.L.; Vainer, B. The morphological growth patterns of colorectal liver metastases are prognostic for overall survival. Mod. Pathol. 2014, 27, 1641-1648. [CrossRef]

20. Fernandez Moro, C.; Bozoky, B.; Gerling, M. Growth patterns of colorectal cancer liver metastases and their impact on prognosis: A systematic review. BMJ Open Gastroenterol. 2018, 5, e000217. [CrossRef]

21. Falcao, D.; Alexandrino, H.; Caetano Oliveira, R.; Martins, J.; Ferreira, L.; Martins, R.; Serôdio, M.; Martins, M.; Tralhão, J.G.; Cipriano, M.A.; et al. Histopathologic patterns as markers of prognosis in patients undergoing hepatectomy for colorectal cancer liver metastases-Pushing growth as an independent risk factor for decreased survival. Eur. J. Surg. Oncol. 2018, 44, 1212-1219. [CrossRef] [PubMed]

22. Baldin, P.; Van den Eynde, M.; Mlecnik, B.; Bindea, G.; Beniuga, G.; Carrasco, J.; Haicheur, N.; Marliot, F.; Lafontaine, L.; Fredriksen, T.; et al. Prognostic assessment of resected colorectal liver metastases integrating pathological features, RAS mutation and Immunoscore. J. Pathol. Clin. Res. 2021, 7, 27-41. [CrossRef] [PubMed]

23. Van den Eynde, M.; Mlecnik, B.; Bindea, G.; Fredriksen, T.; Church, S.E.; Lafontaine, L.; Haicheur, N.; Marliot, F.; Angelova, M.; Vasaturo, A.; et al. The Link between the Multiverse of Immune Microenvironments in Metastases and the Survival of Colorectal Cancer Patients. Cancer Cell 2018, 34, 1012-1026.e1013. [CrossRef] 
24. Mlecnik, B.; Van den Eynde, M.; Bindea, G.; Church, S.E.; Vasaturo, A.; Fredriksen, T.; Lafontaine, L.; Haicheur, N.; Marliot, F.; Debetancourt, D.; et al. Comprehensive Intrametastatic Immune Quantification and Major Impact of Immunoscore on Survival. J. Natl. Cancer Inst. 2018, 110, 97-108. [CrossRef]

25. Brunner, S.M.; Kesselring, R.; Rubner, C.; Martin, M.; Jeiter, T.; Boerner, T.; Ruemmele, P.; Schlitt, H.J.; Fichtner-Feigl, S. Prognosis according to histochemical analysis of liver metastases removed at liver resection. Br. J. Surg. 2014, 101, 1681-1691. [CrossRef] [PubMed]

26. Berthel, A.; Zoernig, I.; Valous, N.A.; Kahlert, C.; Klupp, F.; Ulrich, A.; Weitz, J.; Jaeger, D.; Halama, N. Detailed resolution analysis reveals spatial $\mathrm{T}$ cell heterogeneity in the invasive margin of colorectal cancer liver metastases associated with improved survival. Oncoimmunology 2017, 6, e1286436. [CrossRef]

27. Hoppener, D.J.; Nierop, P.M.H.; Hof, J.; Sideras, K.; Zhou, G.; Visser, L.; Gouw, A.S.; de Jong, K.P.; Sprengers, D.; Kwekkeboom, J.; et al. Enrichment of the tumour immune microenvironment in patients with desmoplastic colorectal liver metastasis. Br. J. Cancer 2020, 123, 196-206. [CrossRef]

28. van Dam, P.J.; Daelemans, S.; Ross, E.; Waumans, Y.; Van Laere, S.; Latacz, E.; Van Steen, R.; De Pooter, C.; Kockx, M.; Dirix, L.; et al. Histopathological growth patterns as a candidate biomarker for immunomodulatory therapy. Semin. Cancer Biol. 2018, 52 Pt 2, 86-93. [CrossRef]

29. Kather, J.N.; Suarez-Carmona, M.; Charoentong, P.; Weis, C.A.; Hirsch, D.; Bankhead, P.; Horning, M.; Ferber, D.; Kel, I.; Herpel, E.; et al. Topography of cancer-associated immune cells in human solid tumors. eLife 2018, 7, e36967. [CrossRef]

30. Yu, J.; Green, M.D.; Li, S.; Sun, Y.; Journey, S.N.; Choi, J.E.; Rizvi, S.M.; Qin, A.; Waninger, J.J.; Lang, X.; et al. Liver metastasis restrains immunotherapy efficacy via macrophage-mediated T cell elimination. Nat. Med. 2021, 27, 152-164. [CrossRef] 